\title{
Amphetamine: Effects of central or systemic injection on hypothalamic activity
}

\author{
HUGH E. CRISWELL \\ Williams College, Williamstown, Massachusetts 01267 \\ and \\ ROBERT A. LEVITT \\ Southern Illinois University, Carbondale, Illinois 62901
}

\begin{abstract}
Dose-response curves showing the effects of amphetamine administration upon multiple unit activity from the lateral hypothalamic area and the ventromedial nucleus of the hypothalamus were obtained. Systemic administration of dextroamphetamine sulfate produced dose-dependent increases in multiple unit activity from both structures, while the direct microinjection of amphetamine into the structures themselves produced a dose-dependent decrease in neural activity from both structures. These data suggest that neither the lateral hypothalamic "feeding center," nor the ventromedial hypothalamic "satiety center," are the neuroanatomical substrate of amphetamine-produced anorexia.
\end{abstract}

Dextroamphetamine (AMP) has been found to produce a wide range of effects on the nervous system. AMP is clinically useful in the treatment of narcolepsy, as an antidepressant, and to improve psychomotor performance (Meyers, Jawetz, \& Goldfien, 1972). The use of AMP in the treatment of hyperactivity has been well documented (Erenberg, 1972), and its use as an appetite suppressant has fostered a multimillion-dollar industry (Brecher, 1972). While these effects appear to result from increased catecholamine release from the presynaptic junction (Meyers, Jawetz, \& Goldfien, 1972), the mode and locus of action of AMP remains somewhat unclear.

The multitude of effects produced by AMP suggest a widespread area of action within the brain; however, individual effects may be more localized. Several investigators have pointed to the hypothalamus as the substrate for the anorexic action of AMP. Two areas within the hypothalamus, the lateral hypothalamic area (LHA) and the ventromedial nucleus (VMN) have been strongly linked to the regulation of food intake and are therefore prime candidates for the site of AMP's anorexic action.

The LHA has been posited to be a "feeding center" with stimulation of this area producing an increase in food intake and lesions of the LHA producing aphagia (Delgado \& Anand, 1953; Epstein, 1960). Similarly, the VMN has been described as a "satiety center" with stimulation of this area producing a decrease in food intake and VMN lesions producing hyperphagia (Anand \& Brobeck, 1951; Epstein, 1960). Recently, these two areas have been implicated in the mediation of amphetamine anorexia (Cole, 1973; Krebs, Bindra, \& Campbell, 1969). In order to determine the effects of

Supported by grants from the National Institute of Mental Health of the United States (MH 14381) and the Southern Illinois University Office of Research and Projects.
AMP upon neural activity within the LHA and VMN, the Krebs group (1969) measured single unit activity from these areas following a systemic injection of an anorexic dose of AMP. They found that systemic AMP resulted in an increase in neural activity within the VMN and a mixed effect upon neural activity within the LHA. Some neurons in the LHA increased their rate of firing following AMP injections and others decreased their rate of firing. These results were described as compatible with either an LHA or a VMN site of amphetamine anorexia. The systemic administration of AMP allows it to effect many brain sites and the changes in neural activity reported by the Krebs group (1969) may have been due to a distal effect of AMP resulting in an indirect effect at the recording site.

The present study examines the effects of both the systemic administration of AMP and the direct microinjection of AMP into the LHA and VMN upon neural activity at these two sites.

\section{METHOD}

\section{Subjects}

Forty-one adult male Long-Evans strain rats, weighing between 400 and $500 \mathrm{~g}$, were maintained in individual cages with food and water available ad lib until the time of surgery. A 12-h light-dark cycle was maintained, and all testing was conducted during the light period.
Surgery
Each rat was deeply anesthetized with sodium pentobarbital (Nembutal, $70 \mathrm{mg} / \mathrm{Kg}$ ) amd placed in a stereotaxic instrument. The scalp was incised and $1-\mathrm{mm}$ burr holes were placed bilaterally, $2 \mathrm{~mm}$ posterior to bregma and $.7 \mathrm{~mm}$ lateral to the midline (VMN placements). Two additional burr holes were placed bilaterally, $3 \mathrm{~mm}$ posterior to bregma and $1.8 \mathrm{~mm}$ lateral to the midline (LHA placements). An array of four chemitrodes was then lowered through the burr holes until the VMN electrodes reached the base of the skull. The electrodes were then raised $.7 \mathrm{~mm}$, placing them at the proper depth for 


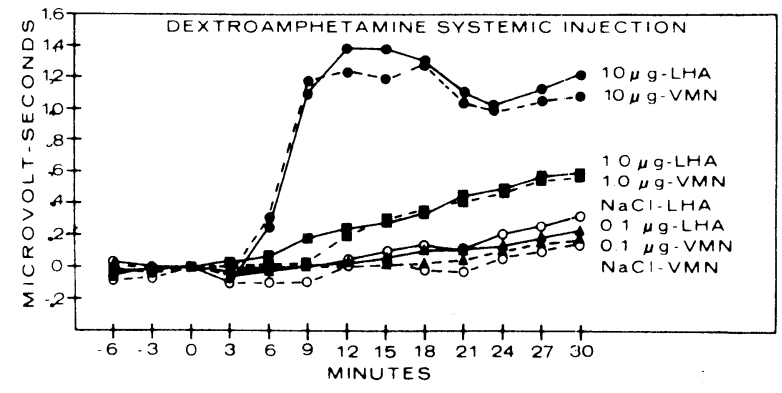

Figure 1. Multiple unit activity changes in the LHA and VMN produced by systemic injections of dextroamphetamine (the drug was injected at time 0 ).

recording, with their tips resting in the bilateral perifornical LHA sites and the bilateral VMN sites. A stainless steel jeweler's screw was placed $4 \mathrm{~mm}$ anterior to bregma on the midline and served as an indifferent electrode.

\section{Electrodes and Recording Equipment}

Microinjections and electrical recording were accomplished by the method previously described by Buerger, Levitt, and Irwin (1973). An array of four chemitrodes consisting of four 30-ga. cannulae insulated except at their tips was lowered into the selected sites. Electrical activity from the tip of each chemitrode was fed into a Grass P17 preamplifier with a lower half-amplitude frequency of $300 \mathrm{~Hz}$ and an upper half-amplitude frequency of $3,000 \mathrm{~Hz}$. This system of filtering eliminates EEG activity, revealing the superimposed spike activity from the population of cells near the tip of the chemitrode (Irwin \& Criswell, 1974). The filtered spike activity was then further amplified and integrated by a Grass 7P3 amplifier-integrator, and the integrated record of multiple unit activity (MUA) was displayed upon a Grass Model 7 polygraph.

\section{Experiment 1}

Following surgical preparation, each subject was transferred from the operating room to an electrically shielded recording room, the chemitrodes were attached by alligator clips to the preamplifiers, and recording was begun. Recording was continued until a steady baseline of neural activity was reached. An on-line calibration signal of $2 \mathrm{MicroV}$ sec was placed across each electrode, and following calibration, a 15-min baseline was recorded at a paper speed of $35 \mathrm{~mm} / \mathrm{min}$. Following the $15-\mathrm{min}$ baseline period, intraperitoneal injections of one of four doses of AMP were administered. The integrated MUA was then monitored for a $30-\mathrm{min}$ postinjection period.

Each drug dosage was administered to five subjects. Doses of dextroamphetamine sulfate were $.1,1.0$, and $10-\mathrm{mg} / \mathrm{Kg}$ body weight. A normal saline control injection was also administered to five subjects. All doses were dissolved in approximately $.4 \mathrm{cc}$ of the saline injection medium. Recordings were obtained from four sites in each animal, producing data from a total of 10 populations of cells for each dose level at each site.

\section{Experiment 2}

The surgical preparation of subjects and the recording methodology of Experiment 2 were identical to that of Experiment 1. The intraperitoneal injection of AMP in Experiment 1 was replaced, however, by the microinjection of one of the four doses of AMP directly into the LHA or VMN through the hollow chemitrode. Following a 1/2-h postinjection period, a second injection was made into the contralateral nonhomologous site. Order of injection was counterbalanced with each site receiving the first injection on half of the occasions. Doses were $.1,1.0$, and 10 micrograms of dextroamphetamine sulfate dissolved in .5 microliters of isotonic saline. Five sites in the LHA and five sites in the VMN received microinjections of the saline control vehicle, three sites in each structure received the .1 microgram dose, five sites in each structure received the 1.0 microgram dose, and eight sites in the LHA and six sites in the VMN received the 10.0 microgram dose.

\section{Histology}

Immediately after the recording session, subjects were perfused with normal saline followed by fixation in $10 \%$ formal saline. Following fixation, 40-micron frozen sections were stained with cresyl violet, and the position of the electrodes was verified. Only subjects with electrodes verified to be within the LHA or the VMN were used in the data analysis.

\section{RESULTS}

\section{Experiment 1}

A dose-response plot of the effects of systemic administration of AMP on MUA from the LHA and VMN as a function of time since the drug injection is shown in Figure 1. An analysis of variance revealed a change in drug action as a function of time $(F=16.02$; $\mathrm{df}=4 / 108 ; \mathrm{p}<.001)$, such that MUA increased following the administration of AMP. There was a differential effect of doses $(\mathrm{F}=18.5 ; \mathrm{df}=2 / 27$; $\mathrm{p}<.001)$. An interaction between dose and time $(\mathrm{F}=6.28 ; \mathrm{df}=8 / 108 ; \mathrm{p}<.001)$ indicated that the differences between the various drug doses increased with time. Scheffe tests indicated that during the 30 -min postinjection period, the $.1 \mathrm{-mg} / \mathrm{Kg}$ group did not differ from the saline control. The $1.0-\mathrm{mg} / \mathrm{Kg}$ group did show significantly greater MUA $(p<.05)$ than the saline control, and the $10.0-\mathrm{mg} / \mathrm{Kg}$ group showed significantly greater MUA $(\mathrm{p}<.05)$ than the $1.0-\mathrm{mg} / \mathrm{Kg}$ group. There were no differences in the drug effects on MUA between the LHA and VMN, and there was no interaction between the recording site and doses in the effect on MUA.

\section{Experiment 2}

When differing doses of AMP were microinjected directly into the LHA or the VMN, no differences in the effect upon MUA were found between the two injection sites, and the site of recording did not interact with time in its effect upon MUA. The data from the two sites were, therefore, combined for the subsequent statistical analyses. The dose-response plot of the effects of microinjection of AMP into the LHA or VMN upon MUA measured from the site of the microinjection is depicted in Figure 2. There was no overall effect of the differences in drug doses employed, but there was an overall decrease in MUA over time $\quad(F=20.44$; $\mathrm{df}=5 / 180 ; \mathrm{p}<.001)$ and an interaction between dose and time $(F=2.36 ; \mathrm{df}=15 / 180 ; \mathrm{p}<.01)$ such that the high doses produced a greater decrease immediately following the microinjection, but this effect disappeared with time. Scheffe tests indicated that during the first 
6-min postinjection period both the 1.0-microgram and the 10-microgram groups showed significantly less MUA than did the saline control $(\mathrm{p}<.05)$, but by the second 6-min period only the 10-microgram group differed significantly from the saline control $(\mathrm{p}<.05)$. In no instance was the .1-microgram group different than the saline control.

\section{DISCUSSION}

The present study does not support a model of amphetamine anorexia based upon a direct action of AMP at either the VMN "satiety center" or the LHA "feeding center." The hypothesis of a differential stimulation or inhibition of neural activity at these sites would predict that both systemically and locally administered AMP should produce a differential response from the two centers. In fact, systemic AMP evoked a similar increase in MUA from each structure, while the direct microinjection of AMP produced a similar decrease in activity at both structures. There was, thus, no evidence of a differential effect of AMP upon the LHA and the VMN.

Even more damaging to a simplistic view of AMP anorexia resulting from the selective excitation or inhibition of the two hypothalamic structures is the data demonstrating that both increases and decreases in neural activity in the LHA or the VMN can be produced by amphetamine in anorexic doses, depending upon the site of administration. If the effect of AMP upon neural activity at the hypothalamic sites is a causal factor in AMP anorexia, then one would not expect an increase in hypothalamic activity following anorexic stimulation in one situation and a decrease in neural activity following anorexic stimulation in another.

While it is possible that subtle changes in the patterns of hypothalamic neural activity which are not detectable by the MUA recording method are responsible for the anorexic effects of AMP, it seems more likely that the anorexic effects of AMP stem from an action upon another site. The recent studies by Gold (1973) and Ahlskog and Hoebel (1973) suggest that the satiety function generally ascribed to the VMN may, in fact, be a function of the ascending ventral noradrenergic bundle described by Ungerstadt (1971). This pathway passes through the LHA as a part of the medial forebrain bundle, and the anorexic action of AMP may be mediated by an effect upon this fiber system rather than by a direct action at the LHA.

\section{REFERENCES}

Ahlskog, J. E., \& Hoebel, B. G. Overeating and obesity from damage to a noradrenergic system in the brain. Science, 1973, 182, 166-168.

Anand, B. K., \& Brobeck, J. R. Hypothalamic control of food intake in rats and cats. Yale Journal of Biology and Medicine, 1951, 24, 123-138.

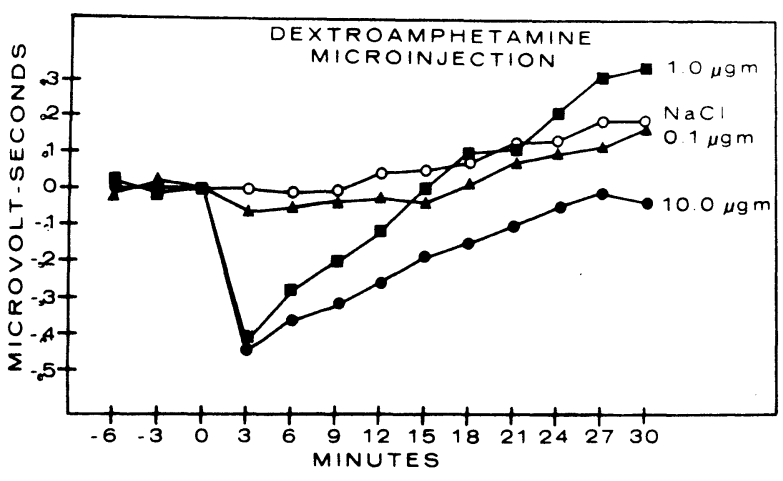

Figure 2. Multiple unit activity changes in the LHA and VMN combined, produced by dextroamphetamine microinjections (the drug was injected at time 0 ).

Booth, D. A. Amphetamine anorexia by direct action on the adrenergic feeding system of rat hypothalamus. Nature, 1968 , 217, 869-870.

Brecher, E. M. Licit and illicit drugs. New York: Little, Brơ̌n, 1972 .

Buerger, P. B., Levitt, R. A., \& Irwin, D. A. Chemical stimulation of the brain: Relationship between neural activity and water ingestion in the rat. Journal of Comparative and Phy siological Psychology, 1973, 82, 278-285.

Cole, S. O. Hypothalamic feeding mechanisms and amphetamine anorexia. Psy chological Bulletin, 1973, 79, 13-20.

Delgado, J. W., \& Anand, B. K. Increase of food intake induced by electrical stimulation of the lateral hypothalamus. American Journal of Physiology, 1953, 172, 162-168.

Erenberg, G. Drug therapy in minimal brain dysfunction: A commentary. Journal of Pediatrics, 1972, 81, 359-365.

Epstein, A. N. Reciprocal changes in feeding behavior produced by intrahypothalamic chemical injections. American Journal of Phy siology, 1960,199, 969-974.

Gold, R. M. Hypothalamic obesity: The myth of the ventromedial nucleus. Science, $1973,182,488-490$.

Irwin, D. A., \& Criswell, H, E. A system for accurate on-line calibration of multiple unit potential recording. Physiology and Behavior, 1974, 12, 703-705.

Krebs, H., Bindra, D., \& Campbell, J. F. Effects of amphetamine on neural activity in the hypothalamus. Physiology and Behavior, 1969, 4, 685-691.

Meyers, F. H., Jawetz, F., \& Goldfien, A. Review of medical pharmacology. Los Altos: Lange Medical Publications, 1972. Teitelbaum, P., \& Epstein, A. The lateral hypothalamic sy ndrome: Recovery of feeding and drinking after lateral hypothalamic lesions. Psychological Reviews, 1962, 69, 74-90.

Ungerstedt, $U$. Stereotaxic mapping of the monamine pathways in the rat brain. Acta Physiologica Scandinavica, 1971, 367, 1-48.

(Received for publication February 22, 1975.) 